\title{
A Low-Complexity Resource Allocation Algorithm for Indoor Visible Light Communication Ultra-Dense Networks
}

\author{
Xiangwei Bai ${ }^{\mathbb{D}}$, Qing $\mathrm{Li}^{*}$ and Yanqun Tang \\ PLA Strategic Support Force Information Engineering University, Zhengzhou 450000, China; \\ bai940102@163.com (X.B.); tangyanqun@126.com (Y.T.) \\ * Correspondence: liqing0206@163.com; Tel.: +86-0371-8162-4122
}

Received: 5 March 2019; Accepted: 29 March 2019; Published: 2 April 2019

check for updates

\begin{abstract}
In this paper, a low-complexity multi-cell resource allocation algorithm with a near-optimal system throughput is proposed to resolve the conflict between the high system throughput and low complexity of indoor visible light communication ultra-dense networks (VLC-UDNs). First, by establishing the optimal model of the resource allocation problem in each cell, we concluded that the problem is a convex optimization problem. After this, the analytic formula of the normalized scaling factor of each terminal for resource allocation is derived after reasonable approximate treatment. The resource allocation algorithm is subsequently proposed. Finally, the complexity analysis shows that the proposed algorithm has polynomial complexity, which is lower than the classical optimal inter-point method. The simulation results show that the proposed method achieves a improvement of $57 \%$ in performance in terms of the average system throughput and improvement of $67 \%$ in performance in terms of the quality of service (QoS) guarantee against the required data rate proportion allocation (RDR-PA) method.
\end{abstract}

Keywords: visible light communication; ultra-dense networks; resource allocation; system throughput; quality of service guarantee

\section{Introduction}

Visible light communication (VLC) is an emerging wireless communication technology, which simultaneously achieves communication and illumination [1,2] and has the advantage of high speeds [3]. Due to the ubiquitous indoor light sources, visible light communication has become an attractive alternative to conventional radio communications for indoor environments [4]. However, the modulation bandwidth of commercial light emitting diodes (LEDs) is only $20 \mathrm{MHz}$ and the bandwidth resource is limited $[5,6]$. Therefore, a key issue in this field is the design of an efficient resource allocation algorithm for visible light communication.

The visible light communication networks are typical ultra-dense networks (UDNs) that have three characteristics: high terminal density, high traffic density requirement and high access point (AP) density. The reference values for the three characters in VLC-UDNs are provided as follows [7]: the terminal density is higher than 0.25 terminals per $\mathrm{m}^{2}$; the traffic density is higher than $10 \mathrm{Mbps} / \mathrm{m}^{2}$; and the distance between the APs is less than $10 \mathrm{~m}$. Being different from the radio signals in the traditional radio frequency ultra-density networks (RF-UDNs), the visible light in VLC-UDNs has a line of sight (LoS) and it is easily blocked, which interrupts communication [8]. The strong directivity of light sources limits the coverage of a single light source and results in the user channel quality being closely related to the terminal's spatial location [9]. Compared with radio frequency networks, VLC-UDNs have a higher AP density, which would cause increase the prominence of interference in 
overlapping areas. It is crucial to design an efficient resource allocation algorithm to overcome these challenges and satisfy the requirements of the terminal.

There are many classical research solutions for the resource allocation problem in multi-cell scenarios for indoor VLC-UDNs. References [10-12] used the Markov chain model to allocate the visible light multi-color channel resources to terminals. Based on the concept of channel reservation, reference [13] proposed an adaptive bandwidth allocation method based on traffic priority. Although reference [10-13] improved the system performance, the algorithm design deviates from the actual spatial scene of VLC-UDNs. References $[14,15]$ allocated resources based on the priority of terminals, which was measured using the fuzzy logic (FL) theory. Although the system throughput and the terminal satisfaction performance were improved, the algorithm complexity increased due to the utilization of centroid method during the defuzzification process. Moreover, the design of fuzzy logic rule table was subjective. References [16-18] used the required data rate proportion (RDR-PA) method to allocate resources. Although the requirements of terminals were considered, the channel quality was ignored and the resource utilization efficiency needed to be improved. Reference [19] used the satisfaction degree proportion method to allocate resources for terminals, but the method was applied only to time resources. Although the algorithms in reference [16-19] had low complexity, the optimal throughput performance was not achieved. Reference [20] used the gradient method to resolve the optimal resource allocation problem and the optimal system throughput was realized. However, when the objective function is a complex nonlinear function, the gradient method has a slow convergence rate. In summary, the optimality of the throughput performance and the low complexity of the resource allocation algorithm are a pair of contradictory indicators. There is the need to design an efficient resource allocation algorithm with optimal throughput and low complexity.

Aiming to resolve the contradiction between optimal throughput performance and low complexity, an efficient resource allocation algorithm is proposed here, which achieves an approximate optimal throughput and quadratic polynomial complexity based on the convex optimization theory. Compared with the classical optimal inter-point method, the proposed algorithm has similar throughput performance and quality of service (QoS) guarantee while reducing the complexity. In the comparisons of system throughput, the proposed algorithm achieves an improvement of $57 \%$ in performance compared to the conventional RDR-PA method and the uniform allocation method [21,22]. In terms of QoS guarantee, the proposed algorithm achieves an improvement of $67 \%$ in performance compared to the conventional RDR-PA method and the uniform allocation method.

This paper is organized as follows. The system model is described in Section 2. The resource allocation algorithm is proposed in Section 3. The simulation results are presented in Section 4. Finally, the conclusions are provided in Section 5.

\section{System Model}

As shown in Figure 1, the indoor visible light communication ultra-dense networks are generated by the following process: (1) $N_{A}$ APs are regularly arranged on the ceiling of the networks. (2) $N_{u}$ terminals are randomly and uniformly distributed on a two-dimensional plane and the vertical distance between the terminals and APs is denoted as $L$. The movement model of terminals adopts the static model or a low-speed movement model. (3) Each cell consists of one AP and terminals that are served by the AP. (4) The visible light transmission channels are approximately considered to be time-invariant channels. 


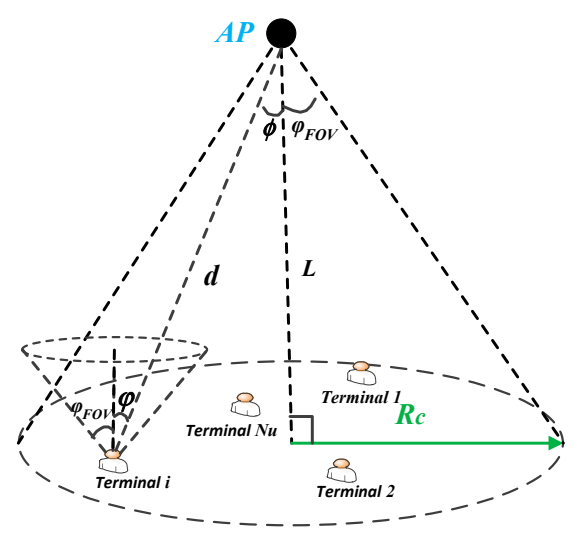

(a)

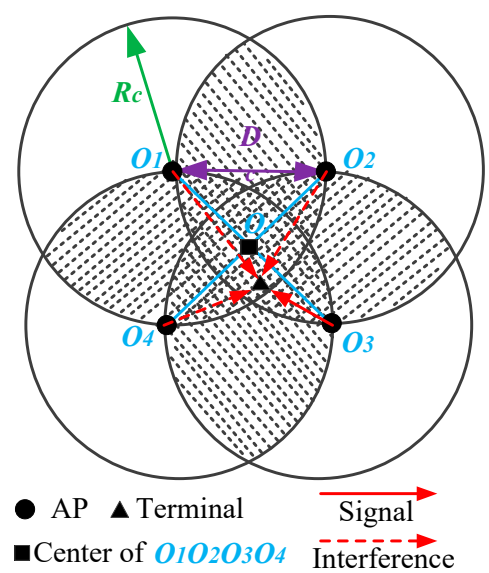

(b)

Figure 1. The visible light communication ultra-dense networks model: (a) the coverage of a single AP and $(\mathbf{b})$ the overlapping areas of a local network.

\subsection{Channel Model}

In the optical line of sight path shown in Figure 1a, the channel gain between the $i$-th terminal and the $j$-th AP is given by [23]:

$$
h_{i, j}=\left\{\begin{array}{l}
\frac{(m+1) A}{2 \pi d_{i, j}^{2}} \cos ^{m}(\phi) T_{S}(\varphi) g(\varphi) \cos (\varphi), 0 \leq \varphi \leq \varphi_{F O V} \\
0, \varphi>\varphi_{F O V}
\end{array}\right.
$$

where $m=-\ln 2 /\left(\ln \left(\cos \phi_{1 / 2}\right)\right)$ is the Lambertian emission order; $\phi_{1 / 2}$ is the half-intensity radiation angle of APs; $A$ is the physical area of the receivers of terminals; $d_{i, j}$ is the distance between the $i$-th terminal and the $j$-th AP; $\phi$ is the angle of irradiance of terminals; $\varphi$ is the angle of incidence of terminals; $\varphi_{F O V}$ is the field of view (FOV) of terminals; $T_{S}(\varphi)$ is the gain of the optical filter; and $g(\varphi)$ denotes the optical concentrator gain, which is formulated as:

$$
g(\varphi)=\left\{\begin{array}{cc}
\frac{n^{2}}{\sin ^{2} \varphi_{F O V}}, & 0 \leq \varphi \leq \varphi_{F O V} \\
0, & \varphi>\varphi_{F O V}
\end{array}\right.
$$

where $n$ is the refractive index. It is assumed that each terminal is served by at most one AP. If the $i$-th terminal is associated with the $\mathrm{AP} j_{a}$, the signal to interference plus noise ratio (SINR) is expressed as follows [24]:

$$
\gamma_{i}=\frac{\left(r P_{t} h_{i, j_{a}}\right)^{2}}{\sum_{j \neq j_{a}}^{N_{A}}\left(r P_{t} h_{i, j}\right)^{2}+n_{0} B_{i, j_{a}}}
$$

where $r$ is the responsivity of receivers; $P_{t}$ is the transmitted optical power of APs; $n_{0}$ is the power spectral density of noise; $B_{i, j_{a}}$ is the bandwidth resource that the AP $j_{a}$ allocates to the $i$-th terminal. The LoS link of the $i$-th terminal might be interrupted by multiple factors, such as blocking. It is assumed that the blocking event random variable obeys the Bernoulli distribution and the probability mass function is given by [25]:

$$
\left\{\begin{array}{l}
P\left(X_{i}=1\right)=1-p_{i} \\
P\left(X_{i}=0\right)=p_{i}
\end{array}\right.
$$

where $X_{i}$ denotes the blocking event random variable of the $i$-th terminal; $X_{i}=1$ indicates that the channel of the $i$-th terminal is not blocked; $X_{i}=0$ indicates that the channel of the $i$-th terminal is blocked; $p_{i}$ denotes the blocking probability of the $i$-th terminal. To reflect the differences among 
terminal channels, it is assumed that the random variable $p_{i}$ obeys the Beta distribution [26]. The achievable data rate of the $i$-th terminal is expressed as follows [27]:

$$
C_{i}=\left(1-p_{i}\right) B_{i, j_{a}} \log _{2}\left(1+\gamma_{i}\right)
$$

The required data rate of the $i$-th terminal, denoted by $R_{i}$, obeys the Gamma distribution [28]. The mathematical expectations of terminal's blocking probability and required data rate are denoted by $\bar{p}$ and $\bar{R}$, respectively.

\subsection{Network Model}

The local network of VLC-UDNs is shown in Figure 1b, which reflects the overlapping relationship among cells. It consists of four regularly arranged cells and indicates the characteristic of high AP density. The coverage radius of a single AP is $R_{c}=L \tan \left(\varphi_{F O V}\right)$. The four APs form the square $\mathrm{O}_{1} \mathrm{O}_{2} \mathrm{O}_{3} \mathrm{O}_{4}$ and $\mathrm{O}$ is the center. Furthermore, $\mathrm{D}_{c}$ denotes the distance between APs. The design of the proposed algorithm is based on the high AP density scenario, which satisfies $D_{c} \leq R_{c}$. When the condition $D_{c} \leq R_{c}$ is satisfied, the seamless illumination coverage is achieved in the VLC-UDNs and each terminal would receive an interference signal.

\section{Resource Allocation Algorithm}

\subsection{Convex Analysis of Resource Allocation Problem}

The signal strength strategy (SSS) is a classical association method for the terminals and APs [16]. Under the SSS association method, a terminal is associated with the closest AP and the multi-cell networks can be equally decomposed into multiple single-cell networks that have interfering signals from neighboring cells. Therefore, if the resource allocation performance of each cell is optimized, the resource allocation performance of the whole network is optimized. The problem of maximizing the throughput under resource constraints for a certain interfering cell is formulated as problem P1:

$$
\begin{aligned}
& \text { P1 : } \max \quad f(\boldsymbol{x})=\sum_{i=1}^{N}\left(1-p_{i}\right)\left(B * x_{i}\right) \log _{2}\left(1+\frac{S_{i}}{I_{i}+n_{0} B x_{i}}\right) \\
& \text { s.t. } \quad x_{i} \geq 0, i=1, \cdots, N \\
& x_{i} \leq 1, i=1, \cdots, N \\
& \sum_{i=1}^{N} x_{i} \leq 1
\end{aligned}
$$

where $x=\left\{x_{1}, \cdots, x_{i}, \cdots, x_{N}\right\} ; x_{i}$ is a certain optimization variable and represents the normalized resource ratio factor that the AP allocates to the $i$-th terminal; $N$ is the general representation of the number of associated terminals in the cell; $B$ is the total bandwidth resource of the cell; $S_{i}$ is the useful signal of the $i$-th terminal; $I_{i}$ is the interfering signal at the $i$-th terminal; and $n_{0} B x_{i}$ is the noise of the $i$-th terminal. Furthermore, the inequality constraints indicate that the normalized resource ratio factor of each terminal ranges from 0 to 1 while the sum of the normalized resource ratio factors is less than 1 . In a certain resource allocation period, if the position of terminals is fixed or the terminals move at an extremely slow speed, $p_{i}, S_{i}$ and $I_{i}$ can be regarded as constants. The noise $n_{0} B x_{i}$ is a function of the optimization variable $x_{i}$. To make our presentation understandable, we define the following two notations: $\alpha_{i}=\left(1-p_{i}\right) B, \beta_{i}=n_{0} B$. The equivalent problem of problem P1 is expressed as:

$$
\begin{array}{lll}
\text { P2: } & \min & f_{0}(x)=\sum_{i=1}^{N}-\alpha_{i} x_{i} \log _{2}\left(1+\frac{S_{i}}{I_{i}+\beta_{i} x_{i}}\right) \\
\text { s.t. } & x_{i} \geq 0, i=1, \cdots N \\
& \sum_{i=1}^{N} x_{i} \leq 1
\end{array}
$$


where $f_{0}(\boldsymbol{x})=-f(\boldsymbol{x})$. The first partial derivative of $f_{0}(\boldsymbol{x})$ with respect to $x_{i}$ is expressed as:

$$
\frac{\partial f_{0}}{\partial x_{i}}=-\alpha_{i} \log _{2}\left(\frac{1+\frac{S_{i}}{I_{i}+\beta_{i} x_{i}}}{e^{\left(\beta_{i}+S_{i}+\beta_{i} x_{i}\right)\left(I_{i}+\beta_{i} x_{i}\right)}}\right)
$$

If we have the notation $g\left(x_{i}\right)=\frac{1+\frac{s_{i}}{I_{i}+\beta_{i} x_{i}}}{e^{\frac{\left.\beta_{i} S_{i}+S_{i}+\beta_{i} x_{i}\right)\left(I_{i}+\beta_{i} x_{i}\right)}{I_{i}}}}$, the second partial derivative of $f_{0}(\boldsymbol{x})$ with respect to $x_{i}$ is expressed as:

$$
\frac{\partial^{2} f_{0}}{\partial x_{i}^{2}}=-\frac{\alpha_{i}}{\ln 2} \cdot \frac{1}{g\left(x_{i}\right)} \cdot \frac{\partial g\left(x_{i}\right)}{\partial x_{i}}
$$

The second mixed derivative of $f_{0}(x)$ is expressed as:

$$
\frac{\partial^{2} f_{0}}{\partial x_{i} \partial x_{j}}=\frac{\partial\left(\frac{\partial f_{0}}{\partial x_{i}}\right)}{\partial x_{j}}=0
$$

As the first partial derivative of $g\left(x_{i}\right)$ with respect to $x_{i}$ is expressed as:

$$
\frac{\partial g\left(x_{i}\right)}{\partial x_{i}}=\frac{-S_{i} \beta_{i}\left(2 I_{i}^{2}+2 I_{i} \beta_{i} x_{i}+2 S_{i} I_{i}+S_{i} \beta_{i} x_{i}\right)}{\left(I_{i}+S_{i}+\beta_{i} x_{i}\right)\left(I_{i}+\beta_{i} x_{i}\right)^{3} e^{\frac{\left.\beta_{i} S_{i} x_{i}+S_{i}+\beta_{i} x_{i}\right)\left(I_{i}+\beta_{i} x_{i}\right)}{\left(I_{i}\right.}}}<0
$$

the maximum and minimum of $g\left(x_{i}\right)$ is expressed as:

$$
\begin{gathered}
g\left(x_{i}\right)_{\max }=\left.g\left(x_{i}\right)\right|_{x_{i}=0}=1+\frac{S_{i}}{I_{i}}>0 \\
g\left(x_{i}\right)_{\min }=\left.g\left(x_{i}\right)\right|_{x_{i}=1}=\frac{1+\frac{s_{i}}{I_{i}+\beta_{i}}}{e^{\frac{\beta_{i} S_{i}}{\left(I_{i}+S_{i}+\beta_{i}\right)\left(I_{i}+\beta_{i}\right)}}}>0
\end{gathered}
$$

According to Equations (11) and (12), we concluded that the second partial derivative of $f_{0}(\boldsymbol{x})$ with respect to $x_{i}$ in Equation (9) is non-negative. Meanwhile, according to Equations (9) and (10), the Hessian matrix of $f_{0}(x)$ is a positive semi-definite matrix or positive definite matrix. Therefore, the objective function of problem P2 is a convex function [29]. In addition, because the inequality constraint functions of problem $\mathbf{P 2}$ are affine functions, the feasibility of problem P2 is related to a convex set [29]. In summary, problem $\mathbf{P} 2$ is a convex optimization problem and the local optimal solution is the global optimal solution [29]. In theory, the global optimal solution can be surely obtained [29].

\subsection{The Proposed Multi-Cell Resource Allocation Algorithm}

If we have the notation $p\left(S_{i}\right)=g\left(x_{i}\right)_{\min }$, the first partial derivative of $p\left(S_{i}\right)$ with respect to $S_{i}$ is expressed as:

$$
\frac{\partial p\left(S_{i}\right)}{S_{i}}=\frac{I_{i}+S_{i}}{\left(I_{i}+\beta_{i}\right) \cdot\left(I_{i}+S_{i}+\beta_{i}\right) e^{\frac{\left.\beta_{i} S_{i}+S_{i}+\beta_{i}\right)\left(I_{i}+\beta_{i}\right)}{\left(S_{i}\right.}}}>0
$$

Therefore, $p\left(S_{i}\right)_{\min }=\left.p\left(S_{i}\right)\right|_{s_{i}=0}=1$ and $g\left(x_{i}\right)_{\min } \geq p\left(S_{i}\right)_{\min }=1$. Combined with Equation (8), the conclusion $\frac{\partial f_{0}}{\partial x_{i}}<0$ can be obtained. Therefore, $\frac{\partial f}{\partial x_{i}}>0$, which means that for a certain terminal, it achieved a higher throughput when it obtains a higher resource ratio. Therefore, the sum of resource ratio factors in a certain cell equaling to 1 is a necessary condition to maximize the throughput performance. The equivalent problem of problem $\mathbf{P} 2$ can be expressed as:

$$
\begin{array}{lll}
\text { P3: } & \min & f_{0}(x)=\sum_{i=1}^{N}-\alpha_{i} x_{i} \log _{2}\left(1+\frac{s_{i}}{I_{i}+\beta_{i} x_{i}}\right) \\
& \text { s.t. } & x_{i} \geq 0, i=1, \cdots N \\
& \mathbf{1}^{T} \boldsymbol{x}=1
\end{array}
$$


Because problem $\mathbf{P} 3$ is a convex optimization problem and it has a feasible solution $x=$ $\left\{x_{i}=\frac{1}{N} \mid i=1, \cdots, N\right\}$, which satisfies the Slater condition, problem $\mathbf{P} 3$ has a strong duality [29]. Because problem $\mathbf{P} 3$ has a strong duality, it satisfies the Karush-Kuhn-Tucher (KKT) condition. By introducing Lagrange multiplier $\lambda_{i}$ for the inequality constraint $x_{i} \geq 0$ and $v$ for the equality constraint $\mathbf{1}^{T} \boldsymbol{x}=1$, the following KKT condition can be obtained [29]:

$$
\left\{\begin{array}{l}
x_{i} \geq 0, i=1, \cdots, N \\
\mathbf{1}^{T} x=1 \\
\lambda_{i} \geq 0, i=1, \cdots, N \\
\lambda_{i} x_{i}=0, i=1, \cdots, N \\
-\alpha_{i} \log _{2}\left(g\left(x_{i}\right)\right)-\lambda_{i}+v=0, i=1, \cdots, N
\end{array}\right.
$$

In Equation (15e), $\lambda_{i}$ is a slack variable. By substituting $\lambda_{i}=v-\alpha_{i} \log _{2}\left(g\left(x_{i}\right)\right)$ into Equations (15c) and (15d), we have:

$$
\left\{\begin{array}{l}
x_{i} \geq 0, i=1, \cdots, N \\
1^{T} x=1 \\
v \geq \alpha_{i} \log _{2}\left(g\left(x_{i}\right)\right), i=1, \cdots, N \\
x_{i}\left(v-\alpha_{i} \log _{2}\left(g\left(x_{i}\right)\right)\right)=0, i=1, \cdots, N
\end{array}\right.
$$

According to Equation (16a), $x_{i}=0$ or $x_{i}>0$. By observing Equation (16d), there are two cases as follows:

i. If $x_{i}=0$, the value of $v-\alpha_{i} \log _{2}(g(0))$ can be an arbitrary real number. Because $g(0)=1+\frac{S_{i}}{I_{i}}$, the value of $v-\alpha_{i} \log _{2}\left(1+\frac{S_{i}}{I_{i}}\right)$ can be an arbitrary real number. Combined with Equation (16.c), we have $v \geq \alpha_{i} \log _{2}\left(1+\frac{S_{i}}{I_{i}}\right)$;

ii. If $x_{i}>0, v-\alpha_{i} \log _{2}\left(g\left(x_{i}\right)\right)=0$. Therefore, $v=\alpha_{i} \log _{2}\left(g\left(x_{i}\right)\right) \geq 0$ and the conclusion satisfies Equation (16c). According to the monotonic decreasing property of $g\left(x_{i}\right)$, we obtained that $v<\alpha_{i} \log _{2}\left(1+\frac{S_{i}}{I_{i}}\right)$. Because $v=\alpha_{i} \log _{2}\left(g\left(x_{i}\right)\right)$, we have $x_{i}=g^{-1}\left(2^{\frac{v}{\alpha_{i}}}\right)$ where $g^{-1}(\cdot)$ is the inverse function of $g(\cdot)$. By defining the notation $\sigma_{i}=\alpha_{i} \log _{2}\left(1+\frac{S_{i}}{I_{i}}\right)$, we have:

$$
x_{i}= \begin{cases}g^{-1}\left(2^{\frac{v}{\alpha_{i}}}\right), & 0 \leq v<\sigma_{i} \\ 0, & v \geq \sigma_{i}\end{cases}
$$

By substituting Equation (17) into Equation (16b), we have:

$$
q(v)=\sum_{i=1}^{N} x_{i}=1
$$

In the left side of Equation (18), $q(v)$ is a piecewise decreasing function with respect to $v$ and the break point is $\sigma_{i}$. Therefore, Equation (18) has a unique solution with respect to $v$ [29]. If the value of $v$ is obtained, the value of $x_{i}$ can be determined by Equation (17) and problem P3 is solved. Because the inverse function of $g\left(x_{i}\right)$ is not an elementary function, we approximate $g\left(x_{i}\right)$ as:

$$
\widetilde{g}\left(x_{i}\right)=\frac{1+\frac{S_{i}}{I_{i}}}{e^{\frac{\beta_{i} S_{i} x_{i}}{I_{I}\left(S_{i}+I_{i}\right)}}}
$$


The inverse function of $\widetilde{g}\left(x_{i}\right)$ is expressed as:

$$
\widetilde{g}^{-1}\left(y_{i}\right)=\frac{I_{i}\left(S_{i}+I_{i}\right)}{\beta_{i} S_{i}} \ln \left(\frac{S_{i}+I_{i}}{I_{i} y_{i}}\right)
$$

The break points of the terminals $\sigma_{i}$ and the lower bound of $v$ form the break point vector $\sigma=\left\{\sigma_{1}, \cdots, \sigma_{i}, \cdots, \sigma_{N}, 0\right\}$. By sorting $\sigma$ in descending order, we obtain another vector $\sigma^{\prime}=$ $\left\{\sigma^{\prime}{ }_{1}, \cdots, \sigma^{\prime}{ }_{i}, \cdots, \sigma_{N}{ }_{N}, 0\right\}$. It is assumed that the value of $v$ is in the $k$-th interval $\left[\sigma^{\prime}{ }_{k+1}, \sigma^{\prime}{ }_{k}\right)$ and the value of $v$ is denoted by $v_{k}$ where $k \in\{1,2, \cdots, N\}$. According to Equations (17), (18) and (20), the approximate value of $v_{k}$ is calculated as follows:

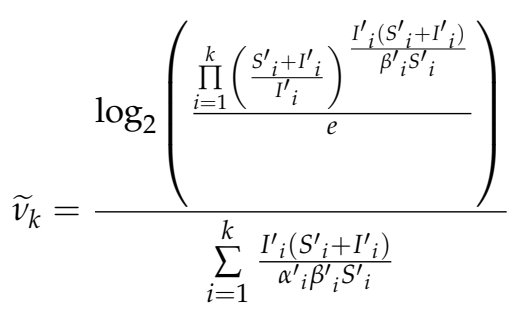

After this, we judge whether the value of $\widetilde{v}_{k}$ is in the interval $\left[\sigma^{\prime}{ }_{k+1}, \sigma^{\prime}{ }_{k}\right)$. If $\widetilde{v}_{k}$ is in the interval $\left[\sigma^{\prime}{ }_{k+1}, \sigma^{\prime}{ }_{k}\right)$, the value of the approximate optimal Lagrange multiplier $\widetilde{v}$ is determined as $\widetilde{v}_{k}$ and the search process is finished. We subsequently calculate the approximate optimal normalized resource ratio factor $\widetilde{x}_{i}$ of each terminal. Otherwise, the judgement process is continued on the $k+1$-th interval and the search process does not quit until $\widetilde{v}$ is in the search interval.

\subsection{Algorithm Description and Asymptotic Complexity Analysis}

The proposed algorithm is summarized as Algorithm 1. The upper bound of the asymptotic complexity depends on the sorting algorithm. The terminal number associated with cell $j$ is denoted as $N_{j}$. When the sorting algorithm adopts the conventional bubble sort algorithm, the operation time of cell $\mathrm{j}$ is $N_{j}^{2}$. Therefore, the total operation time of the whole network Count satisfies:

$$
\begin{aligned}
& \text { Count }=\sum_{j=1}^{N_{A}} N_{j}^{2} \\
& \text { s.t. } \sum_{j=1}^{N_{A}} N_{j}=N_{u}
\end{aligned}
$$

where the constraint condition means that the sum of the terminal number of each cell equals to the terminal number of the whole network. According to the mean inequality theory, the arithmetic mean is less than or equal to the quadratic mean. Therefore, the lower bound of asymptotic complexity can be calculated as:

$$
\text { Count }=\sum_{j=1}^{N_{A}} N_{j}^{2} \geq \frac{\left(\sum_{j=1}^{N_{A}} N_{j}\right)^{2}}{N_{A}}=\frac{N_{u}^{2}}{N_{A}}
$$

If and only if $N_{1}=N_{2}=\cdots=N_{N_{A}}=\frac{N_{u}}{N_{A}}$, the equality holds. According to the inequality theory, the upper bound of the asymptotic complexity can be calculated as:

$$
\text { Count }=\sum_{j=1}^{N_{A}} N_{j}^{2} \leq\left(\sum_{j=1}^{N_{A}} N_{j}\right)^{2}=N_{u}^{2}
$$

If and only if $N_{1}=N_{u}, N_{2}=\cdots=N_{N_{A}}=0$, the equality holds. We denote the lower bound and the upper bound of the asymptotic complexity as $\Omega(\cdot)$ and $O(\cdot)$, respectively [30]. Therefore, the 
lower bound of asymptotic complexity is $\Omega\left(\frac{N_{u}^{2}}{N_{A}}\right)$ and the upper bound of asymptotic complexity is $O\left(N_{u}^{2}\right)$. Because the asymptotic complexity of the conventional inter-point method is $\left(N_{u}^{3.5}\right)$ [31,32], the complexity of the proposed algorithm is lower than the inter-point method.

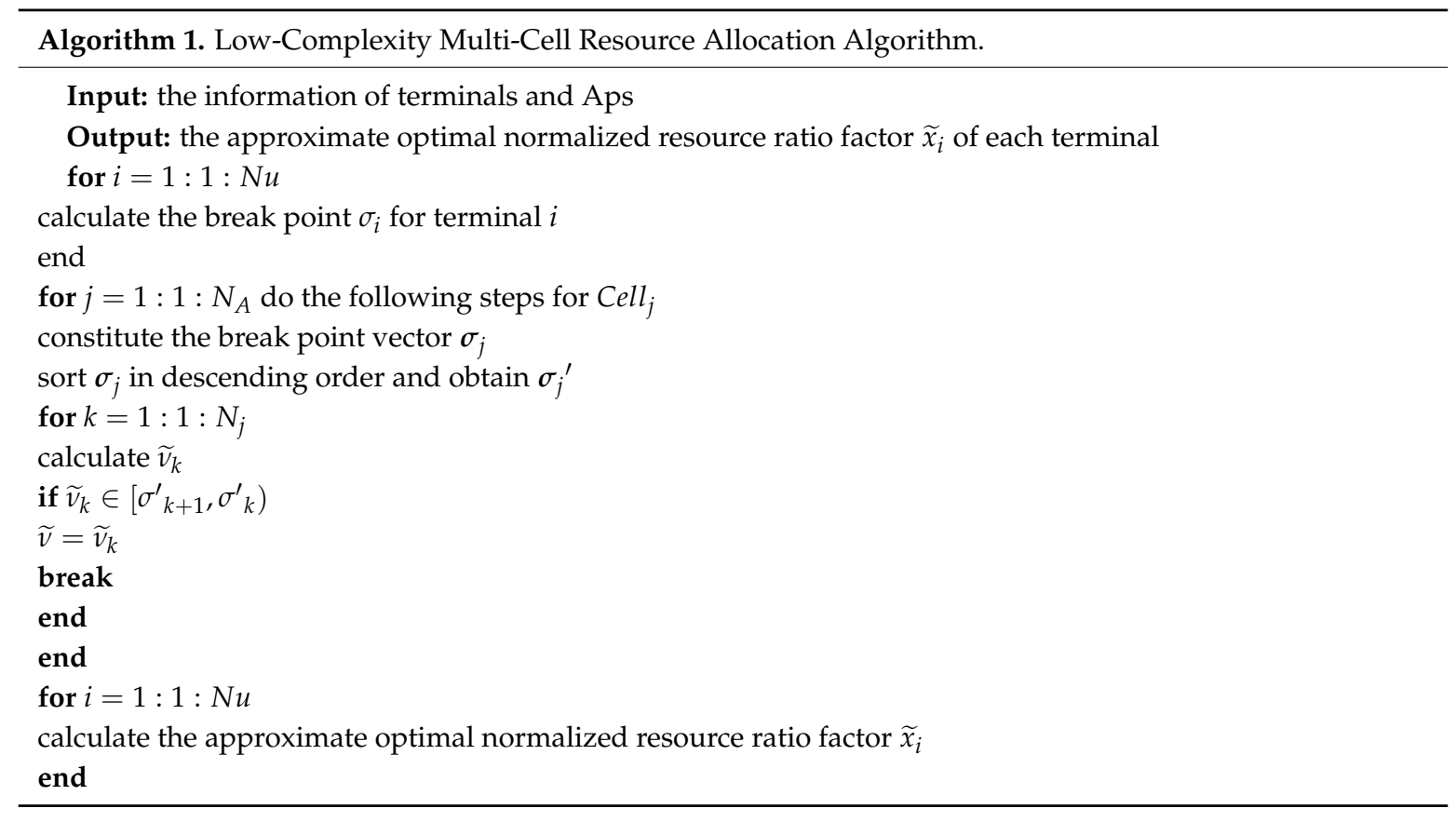

\section{Simulation Results}

\subsection{Conditions}

The Monte Carlo simulations are used to evaluate the performance of the proposed algorithm. Unless specifically stated, the simulation parameters are shown in Table 1. The selected key performance indicators are the system throughput and QoS satisfaction degree. The system throughput is expressed as follows:

$$
C=\sum_{i=1}^{N_{u}} C_{i}
$$

Table 1. Simulation parameters.

\begin{tabular}{cc}
\hline Parameters & Value \\
\hline Room size & $15 \times 15 \times 3 \mathrm{~m}^{3}$ \\
Transmit optical power of AP, $P_{t}$ & $9 \mathrm{~W}$ \\
Vertical distance between the AP and terminal, $L$ & $2.15 \mathrm{~m}$ \\
Half-intensity radiation angle, $\phi_{1 / 2}$ & $60^{\circ}$ \\
FOV of a receiver, $\varphi_{F O V}$ & $60^{\circ}$ \\
The refractive index, $n$ & 1.5 \\
Detector responsivity, $r$ & $0.53 \mathrm{~A} / \mathrm{W}$ \\
The physical area of a receiver, $A$ & $10^{-4} \mathrm{~m}^{2}$ \\
Power spectral density of noise, $n_{0}$ & $10^{-21} \mathrm{~A}^{2} / \mathrm{Hz}$ \\
Bandwidth of each optical AP, $B$ & $40 \mathrm{MHz}$ \\
The gain of the optical filter, $T_{S}(\varphi)$ & 1 \\
Average required data rate, $\bar{R}$ & $40 \mathrm{Mbps}$ \\
Average blocking probability, $\bar{p}$ & 0.1 \\
The access point density, $\rho_{A}$ & $0.11 \mathrm{APs} / \mathrm{m}^{2}$ \\
The terminal density, $\rho_{u}$ & 0.44 terminals $/ \mathrm{m}^{2}$ \\
\hline
\end{tabular}


The QoS satisfaction degree of the $i$-th terminal is given by [14]:

$$
\chi_{i}= \begin{cases}C_{i} / R_{i}, & C_{i}<R_{i} \\ 1, & C_{i} \geq R_{i}\end{cases}
$$

A higher QoS satisfaction degree indicates a better QoS guarantee. The terminal whose QoS satisfaction degree equals to 1 is defined as a satisfied terminal.

We choose the inter-point method in references [31,32], the RDR-PA method in references [16-18] and the conventional uniform allocation method in references $[21,22]$ as comparison methods.

\subsection{Analysis on Terminal Density}

As shown in Figure 2, we study the effect of the terminal density $\rho_{u}$ on the average system throughput. When the terminal density is fixed, the proposed method achieves a similar throughput performance to the inter-point method and outperforms the RDR-PA method and the uniform allocation method. The curves of the four methods follow a similar trend. The average system throughput increases and converges to a certain value for all methods as $\rho_{u}$ increases. However, the growth rate of throughput decreases as $\rho_{u}$ increases. When $\rho_{u}$ is low, the proportion of APs that switch from a non-service status to a service status increases as $\rho_{u}$ increases. Therefore, the average system throughput increases at a significant growth rate. When $\rho_{u}$ reaches a certain threshold, almost all APs are in the service status. Due to the limited resources, the average system throughput converges as $\rho_{u}$ increases. When the average system throughput begins to converge, the terminal density of the proposed algorithm is greater than that of the RDR-PA method and the uniform method, which indicates that the proposed algorithm has a higher resource utilization efficiency and supports higher terminal capacity compared to the other methods. When $\rho_{u}$ is 0.89 , the average throughput of the RDR-PA method is stable at $2.64 \mathrm{Gbps}$, while the average throughput of the proposed algorithm is stable at $4.88 \mathrm{Gbps}$. The performance improvement ratio is $84.8 \%$, which indicates that the proposed algorithm is more suitable for high terminal density scenarios.

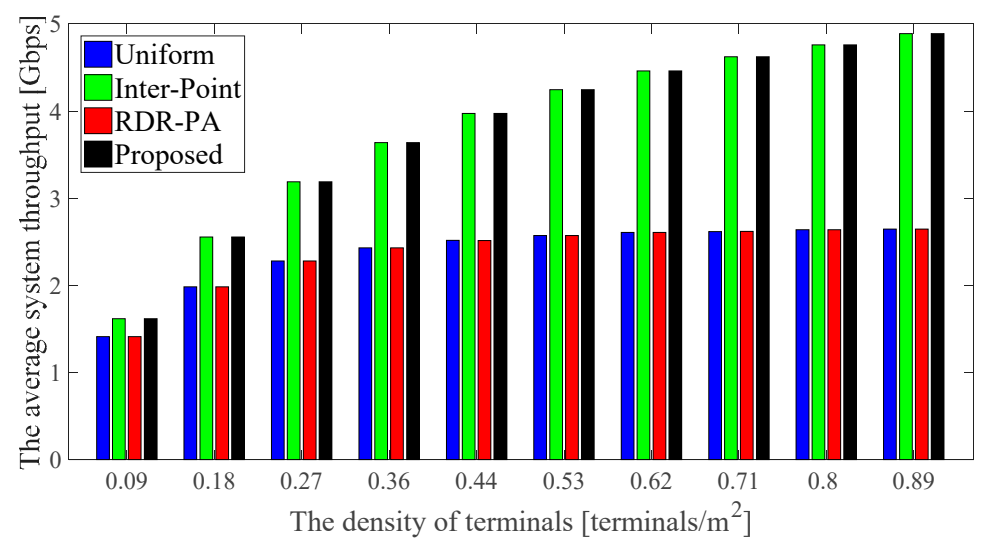

Figure 2. The average system throughput versus the density of terminals.

In Figure 3, the average throughput per terminal is shown as a function of $\rho_{u}$. Figure 3 indicates that an increase in the terminal density will lead to a decrease in the average throughput per terminal. This is because when $\rho_{u}$ increases, the competition for resources among the terminals becomes more intense and the resource allocated to each terminal is relatively reduced. Compared with the RDR-PA method, the performance improvement ratio of the proposed algorithm increases as $\rho_{u}$ increases. When $\rho_{u}$ equals to 0.89 , the performance improvement ratio is $85 \%$.

In Figure 4, the average ratio of satisfied terminals is shown as a function of $\rho_{u}$. In general, the proposed algorithm has a similar performance to the inter-point method and the uniform method outperforms the RDR-PA method. When $\rho_{u}$ equals to 0.22 , the four methods have a similar performance. 
When $\rho_{u}$ is less than 0.22 , the performance of the proposed algorithm is slightly lower than the RDR-PA method and the gap is within $5 \%$. When $\rho_{u}$ is greater than 0.22 , the proposed algorithm outperforms the RDR-PA method and the performance improvement ratio increases as $\rho_{u}$ increases. When $\rho_{u}$ reaches 0.89 , the performance improvement ratio reaches $67 \%$. This is because when $\rho_{u}$ is less than 0.22 , the terminal density is relatively low, the competition among terminals is not intense and the resource is relatively abundant. When $\rho_{u}$ is greater than 0.22 , the competition is more intense as $\rho_{u}$ increases and the advantage of the high resource utilization efficiency of the proposed algorithm is gradually highlighted. The simulation results show that the proposed algorithm has a better QoS guarantee performance in scenarios with high terminal density.

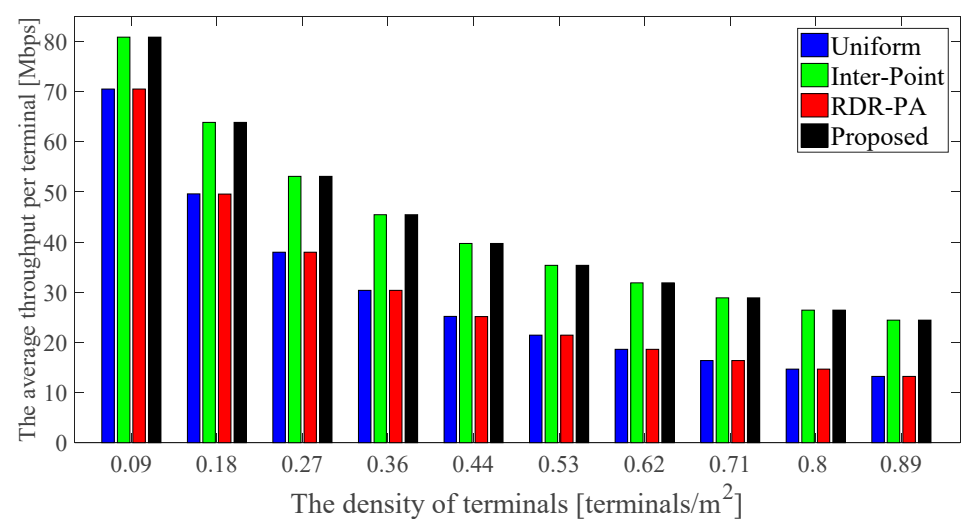

Figure 3. The average throughput per terminal versus the density of terminals.

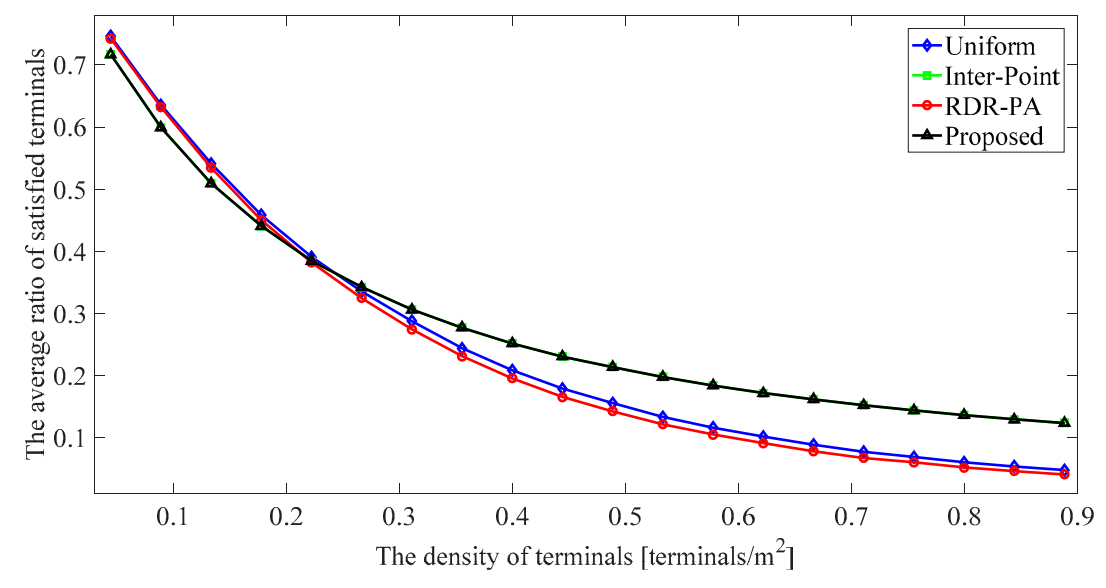

Figure 4. The average ratio of satisfied terminals versus the density of terminals.

\subsection{Analysis on Access Point Density}

As shown in Figure 5, we studied the effect of the AP density $\rho_{A}$ on the average system throughput. The four curves have the same trend, with the average system throughput decreasing as $\rho_{A}$ increases. The proposed algorithm outperforms the RDR-PA method and the uniform method. On one hand, an increase in the AP density increases the total amount of network resources. On the other hand, an increase in the AP density worsens the interference in overlapping areas. In general, the negative effect of interference is greater than the positive effect of the increase resource amount. Therefore, a higher $\rho_{A}$ results in a lower average system throughput. From the perspective of energy saving, the deployment density of APs should be appropriately reduced. Compared with the RDR-PA method, the improvement amount and improvement ratio of average system throughput decreases as $\rho_{A}$ increases. When the AP density is 0.11 , the proposed algorithm achieves the highest throughput improvement of $1.46 \mathrm{Gbps}$ with an improvement ratio of $57.8 \%$. 


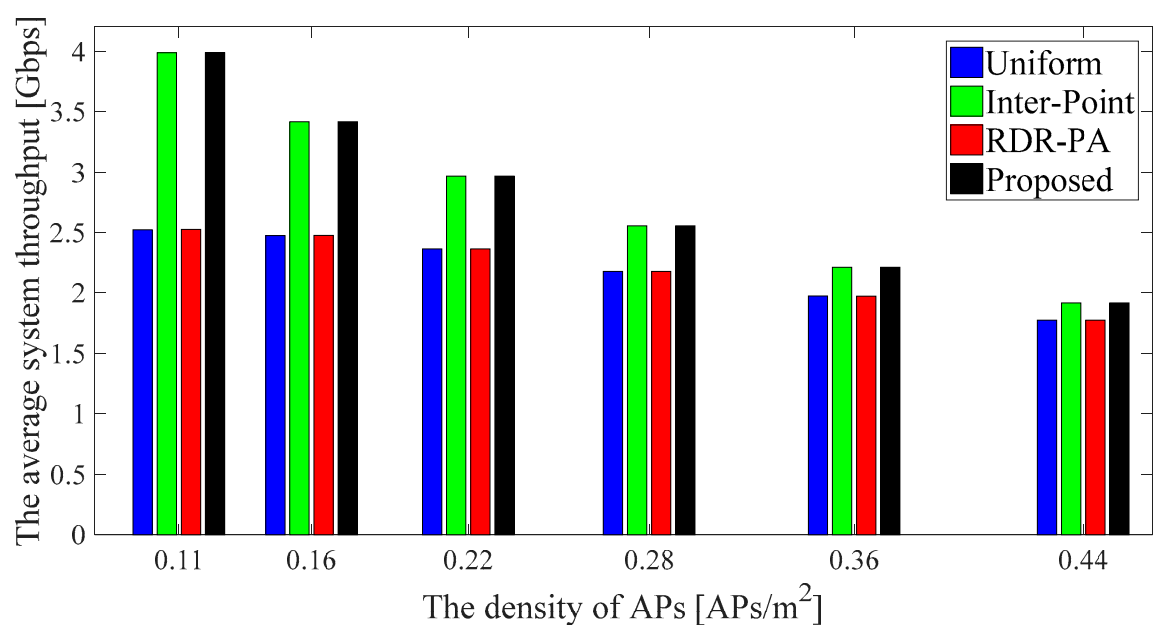

Figure 5. The average system throughput versus the density of access points.

In Figure 6, the average ratio of satisfied terminals is shown as a function of $\rho_{A}$. The performance of the proposed algorithm first increases and then decreases as $\rho_{A}$ increases. When $\rho_{A}$ is 0.25 , the peak value of the proposed algorithm occurs. However, the performance of the RDR-PA method decreases as $\rho_{A}$ increases. This is because the proposed algorithm has a higher resource efficiency. When $\rho_{A}$ is low, the positive effect of an increased resource amount is greater than the negative effect of interference. When $\rho_{A}$ is high, the opposite occurs. When $\rho_{A}$ is 0.22 , the average ratio of satisfied terminals reaches 0.352 . Furthermore, when $\rho_{A}$ is 0.28 , the proposed algorithm achieves the highest performance improvement of 0.222 compared to the RDR-PA method with a performance improvement ratio of $172.8 \%$. In summary, the proposed algorithm has the ability to overcome the inter-cell interference in terms of the average ratio of satisfied terminals.

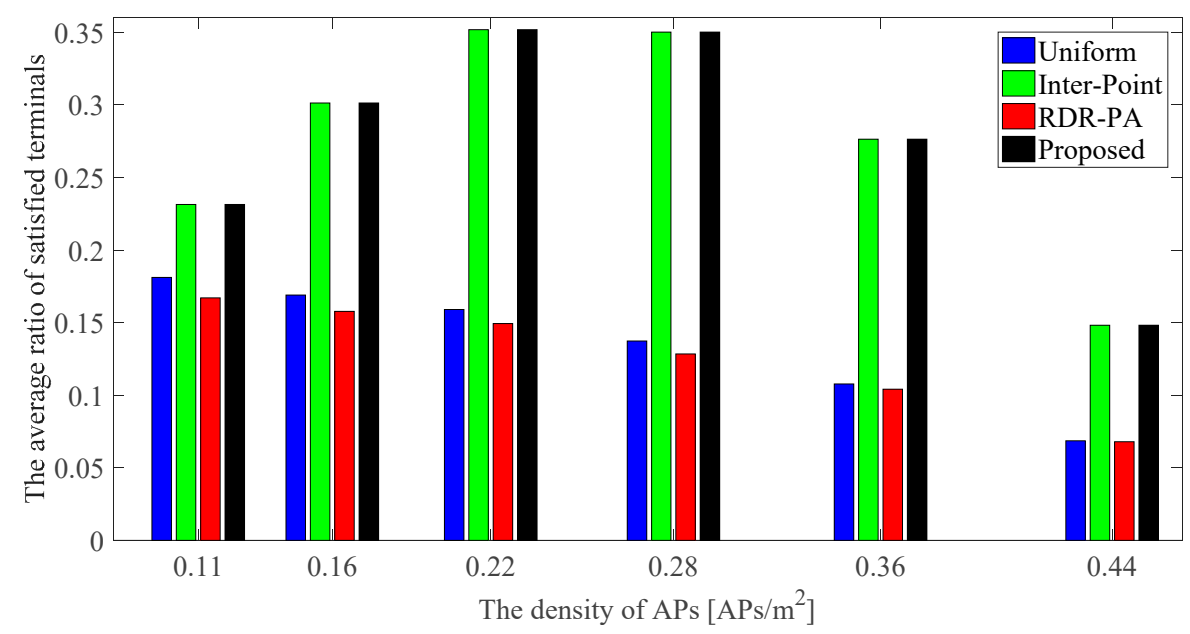

Figure 6. The average ratio of satisfied terminals versus the density of access points.

\subsection{Analysis on Average Required Data Rate}

The effect of the average required data rate $\bar{R}$ on the average system throughput is shown in Figure 7. The performance of the proposed algorithm is stable at $3.97 \mathrm{Gbps}$. This is because the average system throughput is mainly related to the quality of communication channels and $\bar{R}$ has less influence on communication channels. Compared with the RDR-PA method, the proposed algorithm has a performance improvement of $1.45 \mathrm{Gbps}$ with an improvement ratio of $58.0 \%$. 


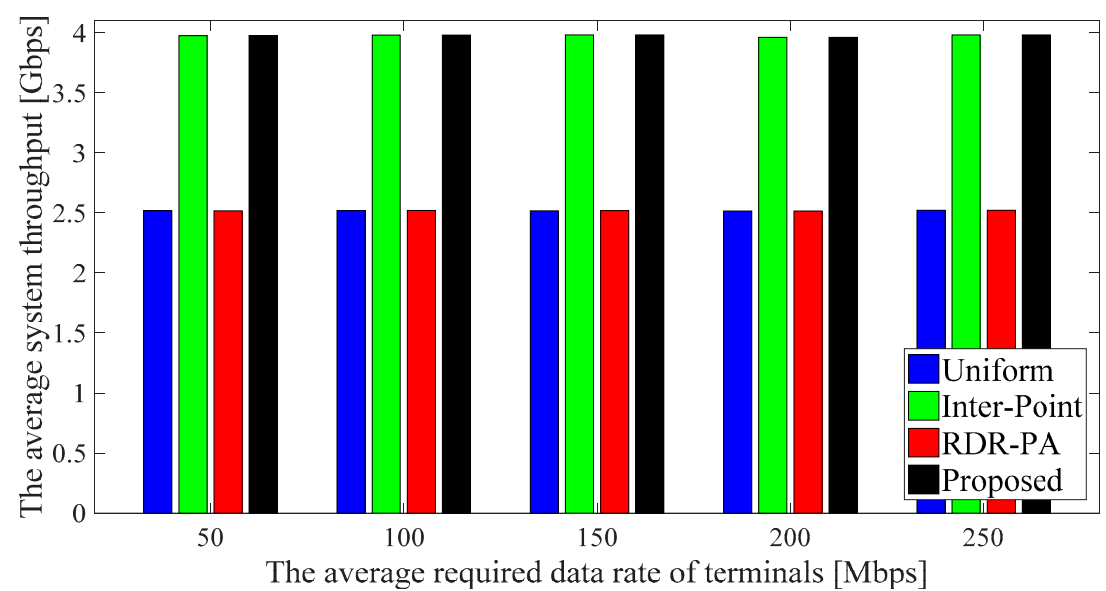

Figure 7. The average system throughput versus the average required data rate of terminals.

In Figure 8, we studied the effect of $\bar{R}$ on the average ratio of satisfied terminals. The performance of the proposed algorithm decreases slowly while the performance of the RDR-PA method decreases rapidly. For example, when $\bar{R}$ increases from $20 \mathrm{Mbps}$ to $100 \mathrm{Mbps}$, the performance of the proposed algorithm decreases from 0.235 to 0.196 and the performance degradation ratio is only $16.9 \%$. In contrast, the performance of the RDR-PA method ranges from 0.447 to 0.024 and the degradation ratio is $94.7 \%$. When $\bar{R}$ is greater than $40 \mathrm{Mbps}$, the proposed algorithm has better performance than the RDR-PA method. This is because the resource shortage become worse as $\bar{R}$ increases and the advantage of the proposed algorithm is highlighted under the high required data rate scenario. When $\bar{R}$ is $100 \mathrm{Mbps}$, the performance of the proposed algorithm is 8.2 times that of the RDR-PA method. The simulation analysis indicates that the proposed algorithm has better QoS guarantee under a high required data rate and is more suitable for scenarios with high traffic requirements.

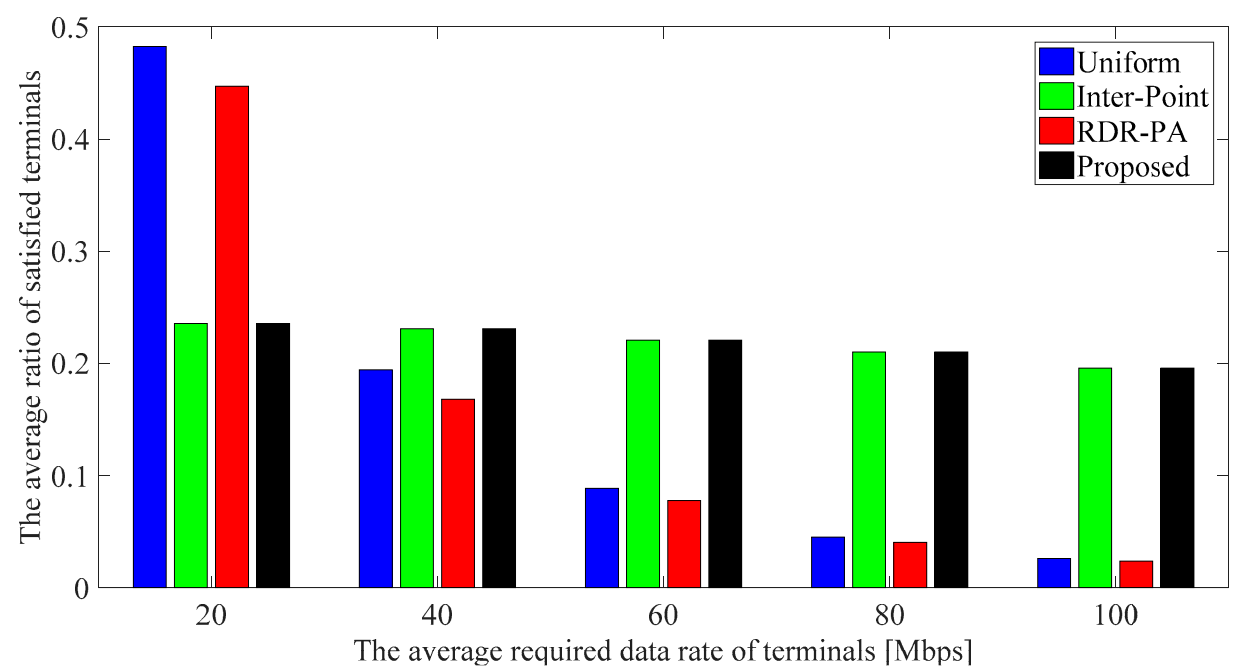

Figure 8. The average ratio of satisfied terminals versus the average required data rate of terminals.

\subsection{Analysis on Average Blocking Probability}

Figure 9 shows the effect of the average blocking probability $\bar{p}$ on the average system throughput. The performance of the proposed algorithm outperforms the RDR-PA method and the uniform method. The performance curves of the four methods follow the same trend. The average system throughput is an approximate negative linear function of $\bar{p}$, which is consistent with Equation (5). Compared with the RDR-PA method, the performance improvement ratio increases as $\bar{p}$ increases and the improvement ratio is at least $56.4 \%$. 


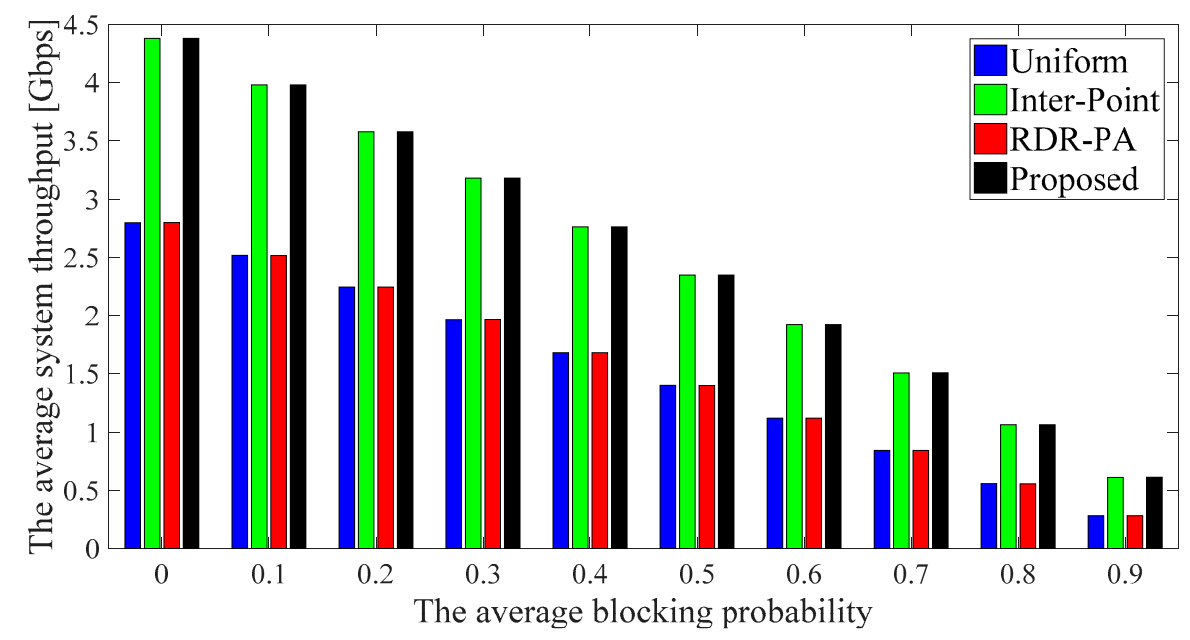

Figure 9. The average system throughput versus average blocking probability.

As shown in Figure 10, we studied the effect of $\bar{p}$ on the average ratio of satisfied terminals. When $\bar{p}$ is fixed, the proposed algorithm outperforms the RDR-PA method and the uniform method. The performance curves of the RDR-PA method and the uniform method gradually decrease as $\bar{p}$ increases, which is similar to a linear decreasing relationship. Compared with the RDR-PA method and the uniform method, when $\bar{p}$ is less than 0.5 , the performance degradation of the proposed algorithm is slower. When $\bar{p}$ is greater than 0.5 , the performance degradation of our method is faster. This is because the resource utilization efficiency of the proposed algorithm is higher compared to the other methods. More specifically, when $\bar{R}$ is fixed, the average achievable data rate is higher than $\bar{R}$ in general if $\bar{p}$ is low. Therefore, the average ratio of satisfied terminals is high and the performance degradation occurs slower. If $\bar{p}$ is high, the quality of communication channels is worse and the performance degradation occurs faster. Compared with the RDR-PA method, the performance improvement ratio increases as $\bar{p}$ increases. When $\bar{p}$ is 0 , the performance improvement ratio is $16.9 \%$. When $\bar{p}$ is 0.1 , the improvement ratio is $39.1 \%$. The simulation results indicate that the proposed algorithm has a better QoS guarantee under different blocking situations.

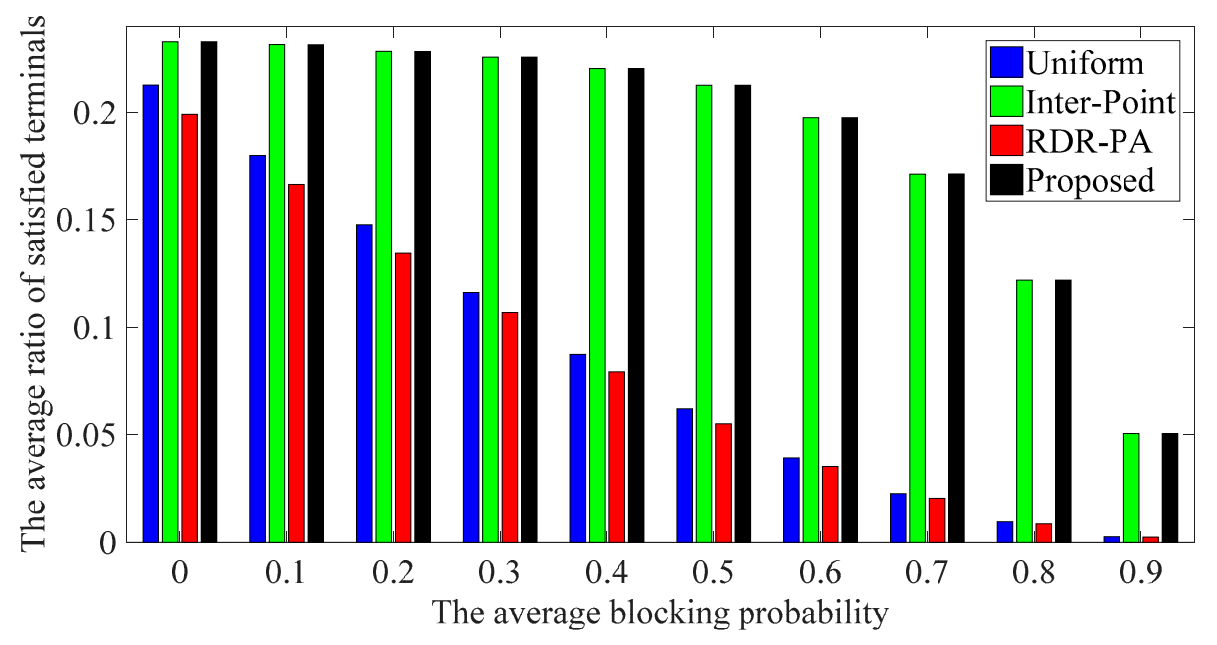

Figure 10. The average ratio of satisfied terminals versus average blocking probability.

\subsection{Analysis on Field of View}

Figure 11 shows the effect of FOV on the average system throughput. To meet the coverage requirements of the proposed algorithm, the deployment density of APs is increased to $0.284 \mathrm{APs} / \mathrm{m}^{2}$. The performance of the four methods decreases as FOV increases. This is because when terminals 
are within FOV, the first derivative of the optical concentrator gain with respect to $\varphi_{F O V}$ satisfies $g^{\prime}\left(\varphi_{F o V}\right)=-2 n^{2} \cos \varphi_{F o V} / \sin ^{2} \varphi_{F o V}$, which is less than 0 according to Equation (2). Therefore, an increase in FOV will lead to a decrease in $g\left(\varphi_{F o V}\right)$. The channel gain will decrease as $g\left(\varphi_{F o V}\right)$ decreases according to Equation (1). Therefore, the FOV angle can be appropriately reduced to improve the average system throughput. However, an excessive reduction of FOV will cause the shrinkage of the coverage area of APs and the formation of communication blind zones. Thus, a tradeoff is needed between the coverage efficiency and throughput performance. The decreasing rates of the four curves decreases as FOV increases. This is because the second derivative of the optical concentrator gain with respect to $\varphi_{F O V}$ satisfies $g^{\prime \prime}\left(\varphi_{F o V}\right)=2 n^{2}\left(1+2 \cos ^{2} \varphi_{F o V}\right) / \sin ^{4} \varphi_{F o V}$, which is greater than 0 . Compared with the RDR-PA method, the performance improvement ratio of the proposed algorithm is stable at $17.2 \%$.

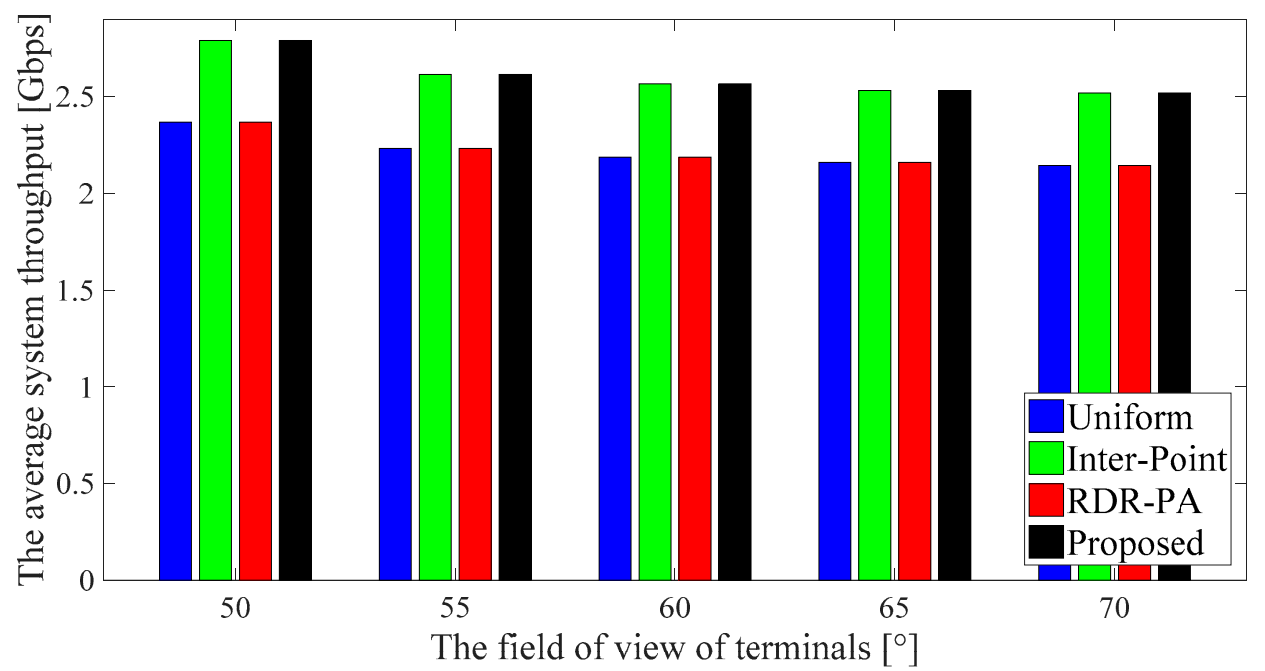

Figure 11. The average system throughput versus the field of view.

In Figure 12, the average ratio of satisfied terminals is shown as a function of FOV. The proposed algorithm has a similar performance to the inter-point method and outperforms the RDR-PA method and the uniform method. The performance decreases as FOV increases. This is because an increase in FOV will lead to a decrease in the average system throughput.

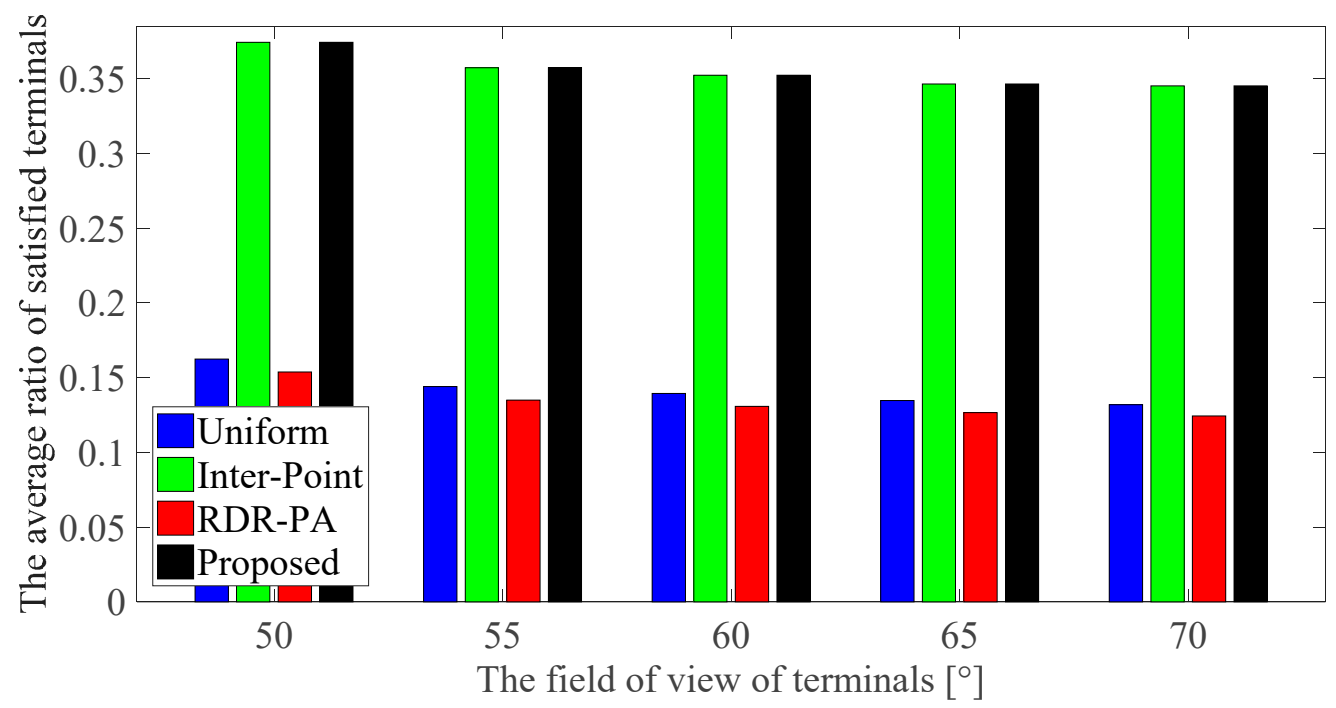

Figure 12. The average ratio of satisfied terminals versus the field of view. 


\subsection{Analysis on Half-Intensity Radiation Angle}

As shown in Figure 13, we studied the effect of the half-intensity radiation angle $\phi_{1 / 2}$ on the average system throughput. The proposed algorithm outperforms the RDR-PA method and the uniform method. The four curves follow the same trend and the average system throughput decreases as $\phi_{1 / 2}$ increases. This is because according to Equation (1), the first derivative of Lambertian emission order $m$ with respect to $\phi_{1 / 2}$ satisfies $m^{\prime}\left(\phi_{1 / 2}\right)=-\ln 2 \cdot \tan \phi_{1 / 2} / \ln ^{2}\left(\cos \phi_{1 / 2}\right)$, which is less than 0 . Therefore, an increase in $\phi_{1 / 2}$ will cause a decrease in $m$ and a reduction in the resource utilization efficiency. Compared with the RDR-PA method, the performance improvement ratio is stable at $57 \%$.

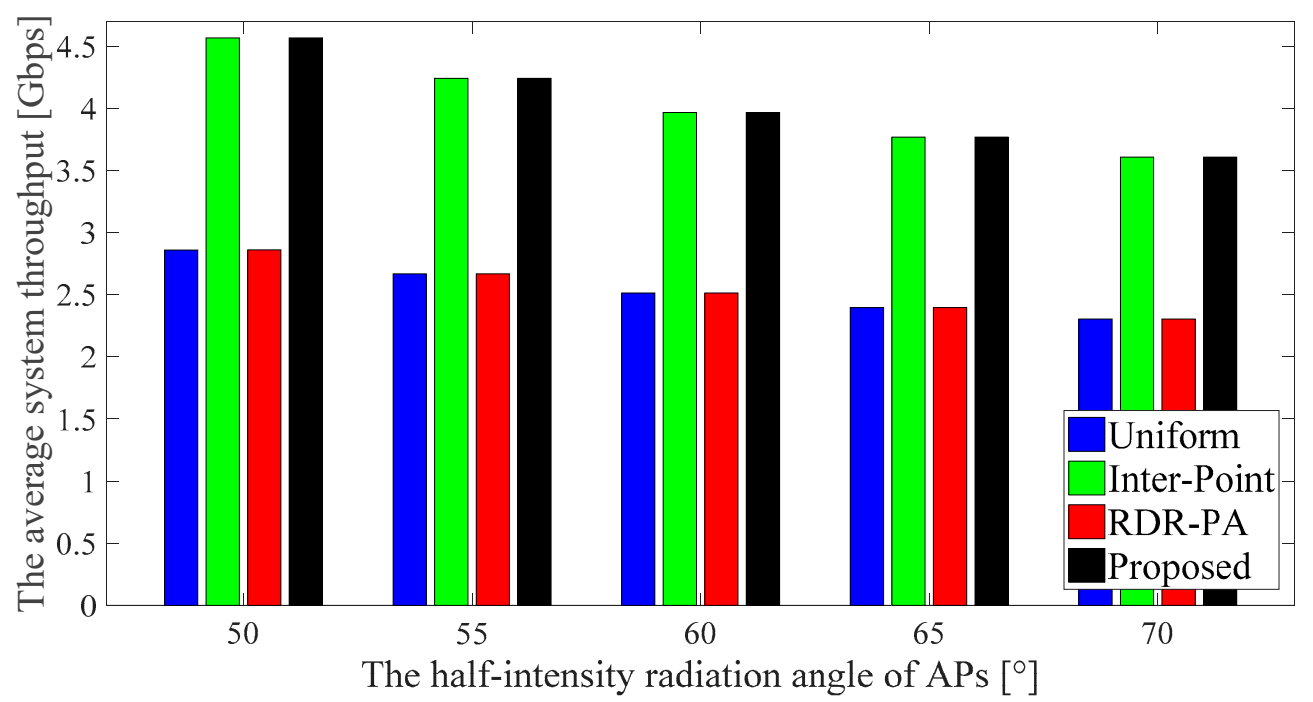

Figure 13. The average system throughput versus half-intensity radiation angle.

In Figure 14, the average ratio of satisfied terminals is shown as a function of $\phi_{1 / 2}$. The performance decreases as $\phi_{1 / 2}$ increases. When $\phi_{1 / 2}$ increases from $50^{\circ}$ to $70^{\circ}$, the average ratio of satisfied terminals of the proposed algorithm decreases from 0.232 to 0.23 with a decrease ratio of only $1 \%$. Furthermore, the performance of the RDR-PA method decreases from 0.208 to 0.14 with a decrease ratio of $32.9 \%$. Compared with the RDR-PA method, the improvement ratio of the proposed algorithm increases from $11.4 \%$ to $64.6 \%$, which indicates the proposed algorithm has a better QoS guarantee.

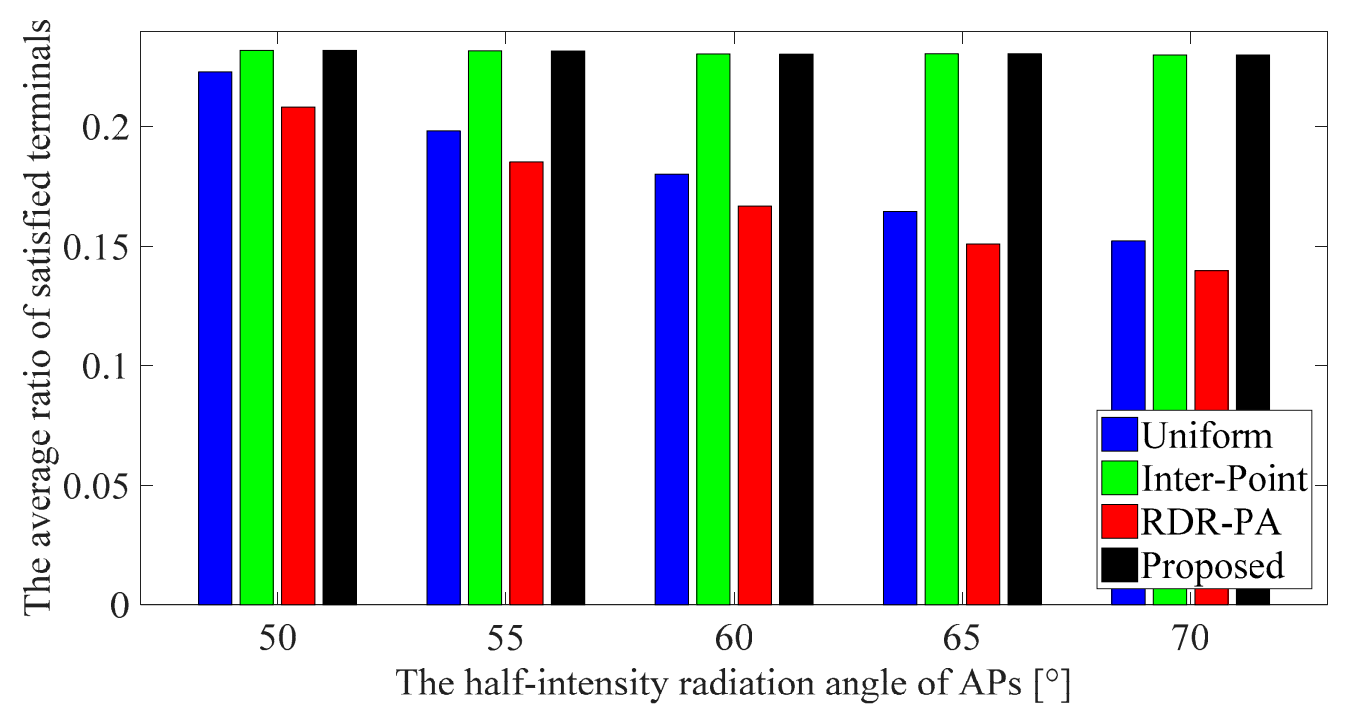

Figure 14. The average ratio of satisfied terminals versus half-intensity radiation angle. 


\section{Conclusions}

In this paper, we proposed a multi-cell resource allocation algorithm with near-optimal system throughput and low complexity based on the convex optimization theory for indoor VLC-UDNs. Compared to the conventional RDR-PA method, the proposed algorithm improves the average system throughput by $57 \%$ and the average ratio of satisfied terminals by $67 \%$. The proposed algorithm is suitable for VLC-UDNs with high terminal density, high traffic density requirement and high access point density. Future research will examine the inter-cell interference cancellation problem and cell virtualization problem.

Author Contributions: Conceptualization, X.B., Q.L. and Y.T.; methodology, X.B. and Q.L.; software, X.B.; investigation, X.B.; resources, Q.L. and Y.T.; writing—original draft preparation, X.B.; funding acquisition, Q.L. and Y.T.; revision work, X.B., Q.L. and Y.T.

Funding: This research was funded by National Natural Science Foundation of China (Grant No.61601516).

Conflicts of Interest: The authors declare no conflict of interest.

\section{References}

1. Li, X.; Jin, F.; Zhang, R.; Wang, J.H.; Xu, Z.Y.; Hanzo, L. Users first: User-centric cluster formation for interference-mitigation in visible-light networks. IEEE Trans. Wirel. Commun. 2016, 15, 39-53. [CrossRef]

2. Zuo, Y.; Zhang, J.; Zhang, Y.Y.; Chen, R.H. Weight threshold check coding for dimmable indoor visible light communication systems. IEEE Photonics J. 2018, 10, 1-11. [CrossRef]

3. Feng, L.F.; Hu, R.Q.; Wang, J.P.; Xu, P.; Qian, Y. Applying vlc in 5g networks: Architectures and key technologies. IEEE Netw. 2016, 30, 77-83. [CrossRef]

4. Haas, H.; Yin, L.; Wang, Y.; Chen, C. What is lifi? J. Lightw. Technol. 2016, 34, 1533-1544. [CrossRef]

5. Chen, C.; Serafimovski, N.; Haas, H. Fractional frequency reuse in optical wireless cellular networks. In Proceedings of the 2013 IEEE 24th Annual International Symposium on Personal, Indoor, and Mobile Radio Communications (PIMRC), London, UK, 8-11 September 2013; pp. 3594-3598.

6. Li, X.; Huo, Y.K.; Zhang, R.; Hanzo, L. User-centric visible light communications for energy-efficient scalable video streaming. IEEE Trans. Green Commun. Netw. 2016, 1, 59-73. [CrossRef]

7. Chen, S.; Qin, F.; Hu, B.; Li, X.; Chen, Z.; Liu, J. User-Centric Ultra-Dense Networks for 5G; Springer: Cham, Switzerland, 2018; p. 7.

8. Li, X.; Zhang, R.; Wang, J.H.; Hanzo, L. Cell-centric and user-centric multi-user scheduling in visible light communication aided networks. In Proceedings of the 2015 IEEE International Conference on Communications (ICC), London, UK, 8-12 June 2015; pp. 5120-5125.

9. Tao, S.Y.; Yu, H.Y.; Li, Q.; Tang, Y.Q. Performance analysis of gain ratio power allocation strategies for non-orthogonal multiple access in indoor visible light communication networks. EURASIP J. Wirel. Commun. Netw. 2018, 2018, 154. [CrossRef]

10. Uddin, M.S.; Chowdhury, M.Z.; Jang, Y.M. Priority-based resource allocation scheme for visible light communication. In Proceedings of the 2010 Second International Conference on Ubiquitous and Future Networks (ICUFN), Jeju Island, Korea, 16-18 June 2010; pp. 247-250.

11. Chowdhury, M.Z.; Uddin, M.S.; Jang, Y.M. Dynamic channel allocation for QoS provisioning in visible light communication. In Proceedings of the 2011 IEEE International Conference on Consumer Electronics (ICCE), Las Vegas, NV, USA, 9-12 January 2011; pp. 13-14.

12. Chowdhury, M.Z.; Uddin, M.S.; Jang, Y.M. Dynamic channel allocation for class-based qos provisioning and call admission in visible light communication. Arab. J. Sci. Eng. 2013, 39, 1007-1016. [CrossRef]

13. Chowdhury, M.Z.; Jang, Y.M.; Haas, Z.J. Priority based bandwidth adaptation for multi-class traffic in wireless networks. Int. J. Multimedia Ubiquitous Eng. 2014, 7, 445-450.

14. Wu, X.; Safari, M.; Haas, H. Bidirectional Allocation Game in Visible Light Communications. In Proceedings of the 2016 IEEE 83rd Vehicular Technology Conference (VTC Spring), Nanjing, China, 15-18 May 2016; pp. 1-5. 
15. Wu, X.; Safari, M.; Haas, H. Three-state fuzzy logic method on resource allocation for small cell networks. In Proceedings of the 2015 IEEE 26th Annual International Symposium on Personal, Indoor, and Mobile Radio Communications (PIMRC), Hong Kong, China, 30 August-2 September 2015; pp. 1168-1172.

16. Wu, X.; Safari, M.; Haas, H. Access point selection for hybrid li-fi and wi-fi networks. IEEE Trans. Commun. 2017, 65, 5375-5385. [CrossRef]

17. Ibrahim, A.; Ismail, T.; Elsayed, K. Optimized radio resource allocation scheme for indoor optical wireless communication. In Proceedings of the 2017 19th International Conference on Transparent Optical Networks (ICTON), Girona, Spain, 2-6 July 2017; pp. 1-4.

18. Wu, X.P.; Basnayaka, D.; Safari, M.; Haas, H. Two-stage access point selection for hybrid vlc and rf networks. In Proceedings of the 2016 IEEE 27th Annual International Symposium on Personal, Indoor, and Mobile Radio Communications (PIMRC), Valencia, Spain, 4-8 September 2016; pp. 1-6.

19. Wang, Y.; Wu, X.; Haas, H. Fuzzy logic based dynamic handover scheme for indoor Li-Fi and RF hybrid network. In Proceedings of the 2016 IEEE International Conference on Communications (ICC), Kuala Lumpur, Malaysia, 22-27 May 2016; pp. 1-6.

20. Kafafy, M.; Fahmy, Y.; Abdallah, M.; Khairy, M. Power efficient downlink resource allocation for hybrid rf/vlc wireless networks. In Proceedings of the 2017 IEEE Wireless Communications and Networking Conference (WCNC), San Francisco, CA, USA, 19-22 March 2017; pp. 1-6.

21. Ye, C.; Gursoy, M.C.; Velipasalar, S. Quality-driven resource allocation for full-duplex delay-constrained wireless video transmissions. IEEE Trans. Commun. 2018, 66, 4103-4118. [CrossRef]

22. Tian, W.; Liu, L.K.; Zhang, X.; Jian, C.X. A resource allocation algorithm combined with optical power dynamic allocation for indoor hybrid vlc and wi-fi network. In Proceedings of the 2016 8th International Conference on Computational Intelligence and Communication Networks (CICN), Tehri, India, 23-25 December 2016; pp. 21-27.

23. Kahn, J.M.; Barry, J.R. Wireless infrared communications. Proc. IEEE 1997, 85, 265-298. [CrossRef]

24. Liu, J.H.; Li, Q.; Zhang, X.Y. Cellular coverage optimization for indoor visible light communication and illumination networks. J. Commun. 2014, 9, 891-898. [CrossRef]

25. Jin, F.; Zhang, R.; Hanzo, L. Resource allocation under delay-guarantee constraints for heterogeneous visible-light and rf femtocell. IEEE Trans. Wirel. Commun. 2015, 14, 1020-1034. [CrossRef]

26. Bai, X.Y.; Li, Q.; Tao, S.Y. Resource allocation based on dynamic user priority for indoor visible light communication ultra-dense networks. In Proceedings of the 2018 IEEE 18th International Conference on Communication Technology (ICCT), Chongqing, China, 8-11 October 2018; pp. 331-337.

27. Kashef, M.; Abdallah, M.; Qaraqe, K. Power allocation for downlink multi-user SC-FDMA visible light communication systems. Am. J. Trop. Med. Hyg. 2015, 77, 390-392.

28. Burchardt, H.; Sinanovic, S.; Bharucha, Z.; Haas, H. Distributed and autonomous resource and power allocation for wireless networks. IEEE Trans. Commun. 2013, 61, 2758-2771. [CrossRef]

29. Boyd, S.; Vandenberghe, L. Convex Optimization; Cambridge University Press: Cambridge, UK, 2004.

30. Graham, R.L.; Knuth, D.E.; Patashnik, O. Concrete Mathematics: A Foundation for Computer Science, 2nd ed.; Addison-Wesley Publishing Company, Inc: Boston, MA, USA, 1994; pp. 443-448.

31. Li, Y.R.; Huang, N.; Wang, J.Y.; Yang, Z.H.; Xu, W. Sum rate maximization for vlc systems with simultaneous wireless information and power transfer. IEEE Photonics Technol. Lett. 2017, 29, 531-534. [CrossRef]

32. Xiao, Y.; Zhu, Y.J.; Zhang, Y.Y.; Sun, Z.G. Linear optimal signal designs for multi-color miso-vlc systems adapted to cct requirement. IEEE Access 2018, 6, 75519-75530. [CrossRef]

(C) 2019 by the authors. Licensee MDPI, Basel, Switzerland. This article is an open access article distributed under the terms and conditions of the Creative Commons Attribution (CC BY) license (http://creativecommons.org/licenses/by/4.0/). 